\title{
Evolution of sex-biased dispersal: the role of sex-specific dispersal costs, demographic stochasticity, and inbreeding
}

Andreas Gros $^{1}$, Thomas Hovestadt ${ }^{1}$, and Hans Joachim Poethke ${ }^{1}$

${ }^{1}$ University of Würzburg, Field Station Fabrikschleichach, Glashüttenstrasse 5, D-96181 Rauhenebrach, Germany

Email addresses:

andreas.gros@biozentrum.uni-wuerzburg.de hovestadt@biozentrum.uni-wuerzburg.de poethke@biozentrum.uni-wuerzburg.de

Postprint version of article published in Ecological Modelling 219 (2008) 226-233 http://dx.doi.org/10.1016/j.ecolmodel.2008.08.014 


\begin{abstract}
Inbreeding avoidance and asymmetric competition over resources have both been identified as factors favouring the evolution of sex-biased dispersal. It has also been recognized that sex-specific costs of dispersal would select for sex-biased dispersal, but there is little quantitative information on this aspect. In this paper we explore (i) the quantitative relationship between cost-asymmetry and a bias in dispersal, (ii) the influence of demographic stochasticity on this effect, and (iii) how inbreeding and cost-asymmetry interact in their effect on sex-specific dispersal. We adjust an existing analytical model to account for sex-specific costs of dispersal. Based on numerical calculations we predict a severe bias in dispersal already for small differences in dispersal costs. We corroborate these predictions in individual-based simulations, but show that demographic stochasticity generally leads to more balanced dispersal. In combination with inbreeding, cost asymmetries will usually determine which of the two sexes becomes the more dispersive.
\end{abstract}

Keywords. asymmetric dispersal costs, kin-selection, numerical model, individualbased simulations 


\section{Introduction}

Dispersal is one of the key processes allowing for the survival of species in fragmented landscapes (Clobert et al. 2001). In the light of the dramatic and rapid changes in our landscapes and of ongoing climatic changes it is thus essential to anticipate how the dispersal abilities and propensities of species evolve in the future. This will require a proper understanding of the mechanisms driving the evolution of dispersal strategies.

Dispersal "decisions" can have far reaching consequences for the fitness of individuals: from founding of new populations to utter failure every outcome of such a decision may occur (Clobert et al. 2001). Considering the vast potential benefits as well as the substantial risks associated with dispersal it is highly plausible that dispersal decisions are context-dependent, i.e. that the decision to disperse is not taken at random but depends on actual environmental conditions, the developmental stage of an individual, and physical condition (Ims and Hjermann 2001; Bowler and Benton 2005; Matthysen 2005; Hovestadt and Nieminen in press). Investigating context-dependent dispersal strategies has thus attracted the interest of both, field biologists and theoretical ecologists.

One obvious kind of context-dependent dispersal, which has gained special interest, is sex-biased dispersal where the propensity to disperse depends on the gender of individuals. Dispersal biased towards males as well as females has both been reported (Clarke et al. 1997; Prugnolle and de Meeus 2002; Lawson Handley and Perrin 2007), but it is not fully understood what factors are responsible for the evolution of sex-biased dispersal as such and especially what determines which of the two sexes is more dispersive (Lawson Handley and Perrin 2007). Yet, two candidate factors favoring gender-biased dispersal have clearly been identified. The first is inbreeding avoidance: its negative consequences can readily be avoided if only one gender disperses because this prevents mating between relatives (Motro 1991, 1994; Gandon 1999; Perrin and Mazalov 1999, 2000). The second is an asymmetry in the intensity in local competition about reproductive ressources (Perrin and Mazalov 2000; Wild and Taylor 2004) where the gender subject to higher competition is dispersing at a higher rate. There is a third and obvious mechanism that should favor the evolution of sex-specific dispersal and that is the presence of an asymmetry in the costs associated with dispersal between the two sexes. This has already, rather parenthetically, been noted by Taylor (1988, p. 368), but he did not explore the topic in any quantitative way. Asymmetry in dispersal costs, however, was also accounted for in the models developed by Leturque and Rousset (2003) and Wild and Taylor (2004). Both models focus on the effect of such an asymmetry on the simultaneous evolution of sex-biased dispersal and offspring sex-ratio, and provide only qualitative statements about the correlation between

sex-specific dispersal costs and the bias in dispersal. In addition, Leturque and Rousset (2003) analyzed a system with habitats that differ in female fecundity, which makes it difficult to isolate the effect of cost asymmetries on model predictions. Furthermore, the analytical models of Taylor (1988), Leturque and Rousset (2003), and Wild and Taylor (2004) ignore the effects of demographic stochasticity, which is known to influence the evolution of dispersal strategies (Ronce 2007). Finally, none of these models investigates the joined influence of inbreeding and cost-asymmetries.

To address these questions we have expanded a model of Gandon (1999) who has 
presented an especially thorough and broad approach to the subject. In his model he accounts for the effects of dispersal costs (but not sex-specific dispersal costs), inbreeding depression and kin-competition, but also of the ploidy-level, control over dispersal decisions (mother or offspring) and population size. Yet, whatever the model configuration, he showed that a certain level of inbreeding depression is necessary to generate sex-biased dispersal. In this paper we will introduce a simple expansion of the analytical model of Gandon (1999) to quantify how slight differences in the costs of dispersal between the two sexes contribute to the evolution of sex-biased dispersal. We will further use an individual-based simulation model (IBM) to quantitatively evaluate the validity of predictions derived from the analytical model under the more realistic condition of finite population size and demographic variability. Finally, we will exemplarily investigate how the joined effect of inbreeding depression and cost asymmetry would affect the evolution of sex-biased dispersal.

\section{Numerical model}

\section{Model description}

We analyze the evolution of dispersal propensity in a meta-population of an annual, sexually reproducing species with discrete generations. Each habitat patch $(i) \operatorname{supports} N$ breeding territories. In all our model variants dispersal is under offspring control. Hence, after hatching, an offspring "decides" whether to disperse or not. Dispersal is sex-specific and is controlled by the dispersers' genotype (one autosomal gene expressed only in males and another - independent one - expressed only in females). In nature dispersal usually incurs costs, such as investment in dispersal mechanism (e.g. flight muscles, longer wings), or an increased probability to die during dispersal. Like Gandon (1999) we subsume all these costs under dispersal mortality $(c)$. Dispersal is at random with respect to which habitat patch is reached (global dispersal). After dispersal, mating and reproduction takes place and the life cycle starts again. In compliance with the model of Gandon (1999), we assume monogamy, i.e. males and females first form breeding pairs by randomly assigning males to females. After pair formation pairs compete for breeding territories. Thus, $N$ pairs - randomly selected from those formed in the previous step - will successfully occupy a territory. Possession of a territory is a prerequisite for reproduction and successful pairs get an equal number of offspring $(\lambda)$. Generations do not overlap. The role of the two sexes is thus completely symmetric. This is important because different intensities of competition for the two sexes may be a prominent factor promoting the evolution of sex-biased dispersal (Perrin and Mazalov 2000, Gros et al., subm.).

Gandon (1999) bases his model on the kin-selection model of Taylor (1988) which without inbreeding depression - makes it possible to calculate the evolutionary stable dispersal probability $d^{*}$ that optimizes inclusive fitness, given certain dispersal costs $(c)$ and a coefficient of relatedness $(R)$ : 


$$
d^{*}=\left\{\begin{array}{lll}
\frac{R-c}{R-c^{2}} & \text { if } \quad R>c \\
0 & \text { if } \quad R \leq c
\end{array}\right.
$$

In the absence of inbreeding depression, this model always predicts symmetric dispersal. We expand this model by introducing sex-specific costs of dispersal for females $\left(c_{f}\right)$ and males $\left(c_{m}\right)$ into the calculation of the coefficient of relatedness $(R)$ and the calculation of the proportion of surviving dispersers. In this case, the modified (omitting inbreeding depression) model predicts different dispersal probabilities for the two sexes:

$$
d_{f}^{*}=\left\{\begin{array}{lll}
\frac{R-c_{f}}{R-c_{f}^{2}} & \text { if } & R>c_{f} \\
0 & \text { if } & R \leq c_{f}
\end{array}\right.
$$

and to:

$$
d_{m}^{*}=\left\{\begin{array}{lll}
\frac{R-c_{m}}{R-c_{m}^{2}} & \text { if } \quad R>c_{m} \\
0 & \text { if } \quad R \leq c_{m}
\end{array}\right.
$$

In the Appendix we present a more detailed derivation of these equations.

Relatedness $(R)$ is - with offspring control of dispersal (comp. Ro in Gandon 1999) calculated as follows:

$$
R=\frac{1}{2 N-k_{1}-((N-1) / 2)\left(2 k_{1}+k_{2}\right)^{2}}
$$

with

$$
\begin{aligned}
k_{1} & =\frac{\left(1-d_{m}\right)\left(1-d_{f}\right)}{\left(1-c_{m} d_{m}\right)\left(1-c_{f} d_{f}\right)} \\
k_{2} & =\frac{\left(1-d_{m}\right)\left(1-c_{f}\right) d_{f}+\left(1-d_{f}\right)\left(1-c_{m}\right) d_{m}}{\left(1-c_{m} d_{m}\right)\left(1-c_{f} d_{f}\right)}
\end{aligned}
$$

As $R$ recursively depends on dispersal rates, it is not possible to solve equations 2 and 3 analytically. We thus found numerical solutions for the evolutionary stable dispersal strategies using Mathematica 4.0 (Wolfram Research, Inc. 1999) by letting the system of $R, d_{f}$ and $d_{m}$ iterate until equilibrium. This is done by first calculating $R$, from which we can calculate $d_{m}$ and $d_{f}$, from which we can again calculate $R$. This is iterated until the rate of change for $d_{m}$ drops below $10^{-4}$.

The numerical model readily allows analyzing the factors influencing the system's behavior. Thus, the effect of the relative differences in dispersal costs $\Delta_{c}=\left(c_{f}-c_{m}\right) /\left(\left(c_{f}+\right.\right.$ $\left.c_{m}\right) / 2$ ) and of the number of breeding pairs $(N)$ on the evolutionary stable dispersal strategies $\left(d_{f}^{*}, d_{m}^{*}\right)$ can be calculated directly. We exemplarily use all combinations of three values for $\Delta_{c}: \Delta_{c} \in\{0.02,0.05,0.10\}$ and three values for $N:(N \in\{10,20,50\})$ to demonstrate the effect of $N, \bar{c}=\left(c_{f}+c_{m}\right) / 2$ with $0.0 \leq \bar{c}<1.0$, and $\Delta_{c} \in\{0.02,0.05,0.10\}$ 
on predicted dispersal probabilities and the magnitude of sexual bias in dispersal. Without loss of generality, we always assume that dispersal is less costly for males than females. In the opposite case results would be mirror images of the ones presented here.

\section{Numerical results}
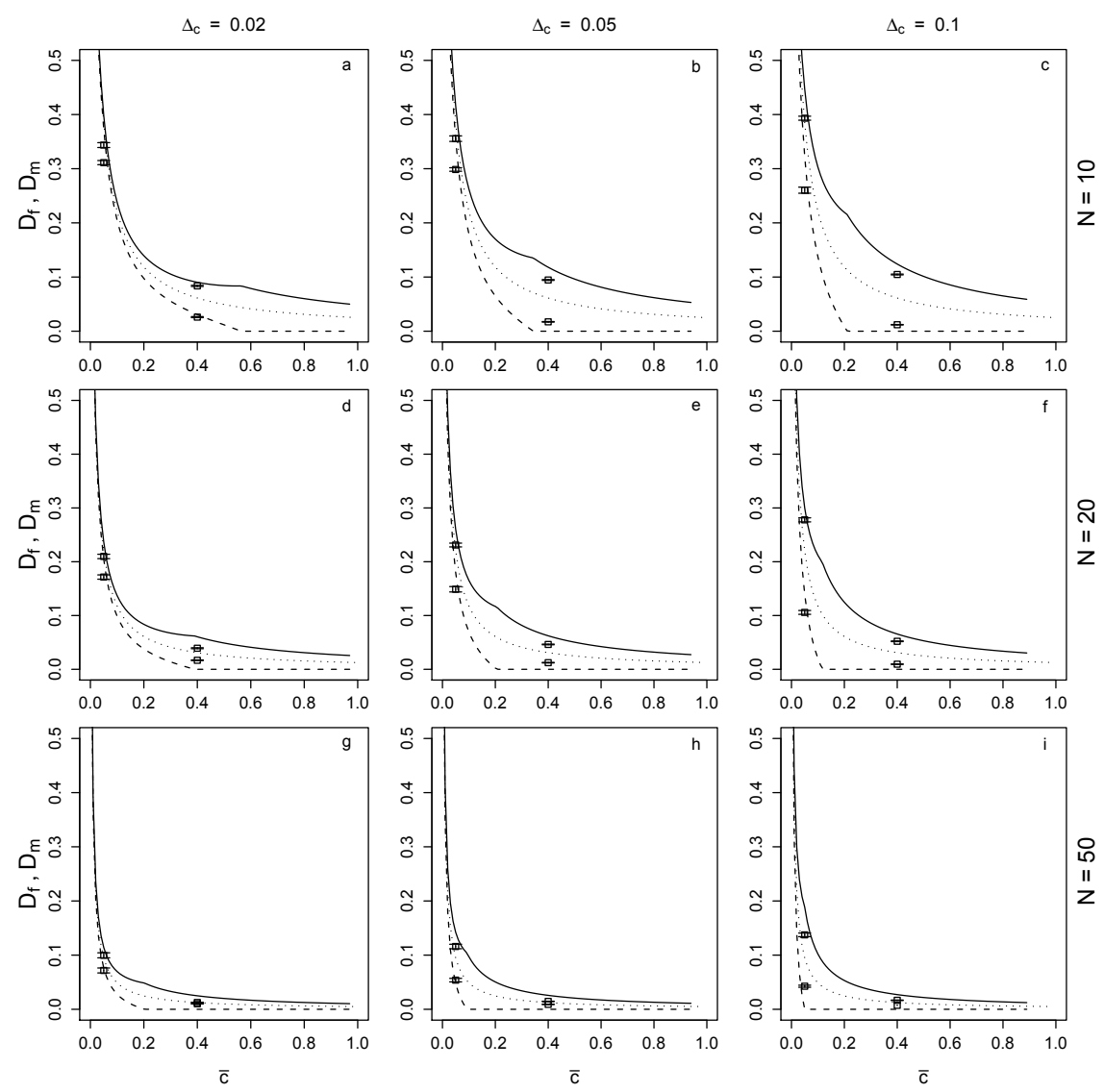

Figure 1: Evolutionarily stable emigration probabilities $\left(d_{f}, d_{m}\right)$ as a function of mean dispersal costs for different values of breeding territories per patch $(N \in\{10,20,50\})$ and for different relative differences in dispersal cost $\left(\Delta_{c} \in\{0.02,0.05,0.10\}\right)$. Lines give predictions based on numerical results, points with error bars give those emerging in individual-based simulations. Continuous lines: male dispersal; dashed lines: female dispersal; dotted lines: result of the numerical solution for $\Delta_{c}=0$, i.e. equal costs of dispersal for both sexes. The noticeable glitches in the curves for male dispersal result from the bounding of female dispersal to zero (eq. 2). The dashed and broken lines end where female dispersal mortality $c_{f}=1.0$. Within rows the dotted lines $\left(\Delta_{c}=0\right)$ are all identical, contrary to superficial visual impression.

Monogamy makes the roles of the two sexes absolutely symmetric. Thus, without inbreeding depression no differences in the dispersal propensity of the sexes are predicted as long as the costs of dispersal are identical for the two sexes. The dotted lines in Figure 1 comply with the results of Gandon (1999) and show the symmetric ESS predicted in case of no difference in dispersal mortality $\left(\Delta_{c}=0\right)$. However, the numerical results clearly 
show that already small cost differences may lead to dispersal strongly biased towards the sex with lower costs (Figure 1). With increasing costs the bias becomes stronger and reaches a maximum exactly at the moment where the predicted dispersal probability first reaches zero for the less dispersive sex (females), i.e. where $R=c_{f}$. Evidently, the predicted difference in dispersal probability increases as the difference in dispersal costs $\left(\Delta_{c}\right)$ increases.

At a given mean cost of dispersal and a fixed level of cost-asymmetry, the sex-bias in dispersal becomes more pronounced with increasing population size (this can be deduced from the left-wards shift of the "glitch" in male and female dispersal probability as patch capacity becomes larger in Figure 1). This can readily be explained by the declining importance of kin-competition - a factor favoring symmetric dispersal - as population size increases (Perrin and Mazalov 2000).

\section{Individual-based simulations}

\section{Model description}

The numerical model ignores the potential influence of demographic stochasticity, which can have a strong effect on the evolution of dispersal probabilities, especially if population size is small and growth rates are low (Parvinen et al. 2003). We thus compare the numerical results (as shown in Figure 1) to results of simulation experiments generated with an individual-based simulation model (IBM). We use a modified version of the individual-based metapopulation model for the evolution of dispersal propensity of an annual, diploid, and sexually reproducing species with non-overlapping generations more thoroughly described in Poethke and Hovestadt (2002). Simulation experiments are initialized by placing $2 N$ individuals in each patch. Note that we do not need to explicitly introduce kin-competition into our simulations as it emerges by default in any individual-based simulation (Poethke et al. 2007). As demographic effects and the role of kin-competition become less prominent with increasing population size, we use different numbers of breeding territories per patch to investigate the effects of demographic stochasticity on the evolutionary outcome. To keep the number of individuals in the whole metapopulation comparable (approximately 25000 breeding individuals) we modified the number of local populations accordingly. Thus, the meta-population consists of $16 \times 16$, $26 \times 26$ and $36 \times 36$ habitat patches with a local number of breeding territories $(N)$ of 50 , 20 and 10 , respectively.

Like in the analytical model, a breeding territory represents a chance to reproduce. We distinguish between patches, but the patches themselves have no other attribute than size, i.e. the number of breeding territories. As in the numerical model we assume that males and females are monogamous and form pairs. Breeding pairs are formed by randomly assigning males to females. Pairs undergo contest competition for each territory: we draw a pair randomly until either all pairs have received a territory or all territories are occupied. A pair can only occupy one territory. Thus, at most $N$ pairs successfully acquire a breeding territory. Each of these then gets $\lambda$ offspring with sex allocated randomly. All 
offspring survive and compete for mates and breeding territories after optional dispersal.

Each individual is characterized by four alleles $\left(m_{1}, m_{2}, f_{1}, f_{2}\right)$ at two diploid loci $(m, f)$, inherited from its parents. The first of these loci $(m)$ determines a male's propensity for dispersal $\left(d_{m}\right)$, the second $(f)$ determines $d_{f}$ in females. The alleles of these loci can take continuous values between 0 and 1 . When inheriting a parent's genes, each allele transferred to the offspring mutates with probability 0.002: affected alleles are altered by adding a random value drawn from a uniform distribution within the interval [-0.02, 0.02]. To check for the influence of the initial conditions on our results we choose a broad range of initial values for $m_{i}$ and $f_{i}$. Individuals are initialized as homozygote at both loci with $m_{i}=0.20-f_{i}$ and $f_{i}$ randomly chosen from $(0.00,0.02,0.04,0.06 \ldots 0.20)$.

We run 50 simulation experiments for each combination of the 11 start values for $f_{i}, m_{i}$ (as described above), the three values of $N: N \in\{10,20,50\}$, two values of $\bar{c}: \bar{c} \in\{0.05,0.4\}$, and three values of relative differences in dispersal costs $\left(\Delta_{c} \in\right.$ $\{0.02,0.05,0.1\})$. We thus run a total of $50 \times 11 \times 3 \times 2 \times 3=9.900$ simulations. The number of offspring was fixed to $\lambda=10$ (but see discussion) in all simulation runs. To allow the populations to reach evolutionary equilibrium, we let simulations run for 50.000 generations. However, preliminary runs showed that equilibrium was usually reached much faster. We record the resulting values for the loci, as well as all other measures mentioned below in the gamete phase in the last generation.

\section{Results of individual-based simulations}

Without inbreeding depression, simulation runs always converge to (approximately) the same final trait distribution independent of the values used for initializing $d_{m}$ and $d_{f}$ (Figure 1). It is always the sex with lower costs of dispersal (males) which evolves higher dispersal probability even if simulations were initialized with female biased dispersal. The results of the simulation runs fundamentally agree with the predictions derived from the numerical model. Most importantly, also in the simulations asymmetric dispersal probabilities evolve already with very slight differences in dispersal costs: Even for a relative difference in dispersal cost of $\Delta_{c}=0.02$ the sex with less costs clearly dominates in dispersal and for $\Delta_{c} \geq 0.05$, sufficiently high mean dispersal cost $(\bar{c} \geq 0.4)$ may result in an nearly complete bias with only one dispersing gender. In this respect our findings (which also emerge in the numerical model) differ from Gandon's who found that an increase in the mean cost of dispersal reduces the magnitude of the sexual bias in dispersal in the case of inbreeding depression. However, the magnitude of sex-bias consistently remains below that predicted by the numerical model especially when the predicted mean dispersal probability becomes small, e.g. for $N=50$ (Figure 1).

For the case that inbreeding depression exists, Gandon (1999) already predicts completely biased dispersal (under the assumption that demographic stochasticity can be neglected), yet that there are always two evolutionary stable equilibria. Which of the two sexes will become the dispersive one should depend on the initial conditions, only. To investigate how the emergence of this bi-stability is affected by the presence of a costasymmetry we performed simulations with different combinations of initial male $\left(d_{m}\right)$ and 

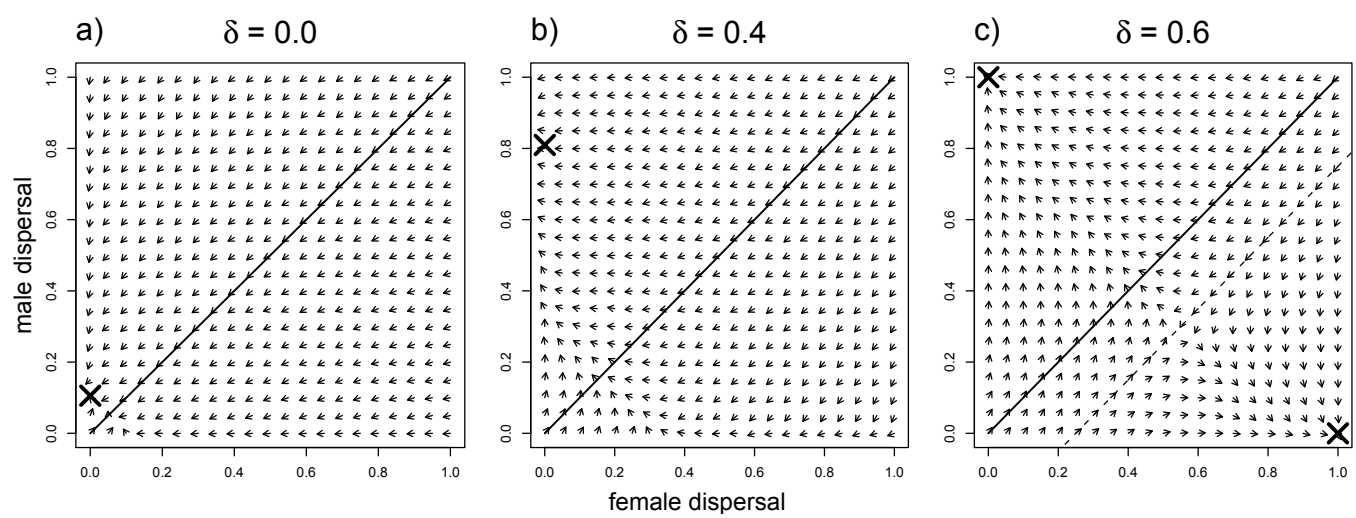

Figure 2: Vectorfields and evolutionarily stable points for different values of inbreeding depression: $\delta=0.0(\mathrm{a}), \delta=0.4(\mathrm{~b}), \delta=0.6$; the panels show the resulting evolutionary trajectories over 1000 generations (averaged over ten replicate simulations) in the $d_{f}-d_{m}$-space and the evolutionarily stable results of simulations running over 50.000 generations marked with $\mathrm{X}$. Parameters used: $N=10, \bar{c}=0.4$, and $\Delta_{c}=0.1$.

female $\left(d_{f}\right)$ dispersal probabilities spanning the whole surface of the possible parameter space. The resulting evolutionary trajectories (over 1000 generations) in the $d_{f}-d_{m}$-space were averaged over ten replicate simulations with identical initial values for $d_{f}$ and $d_{m}$. Inbreeding depression is implemented in the same way as in Gandon (1999): whenever a female and a male, which both did not disperse, form a pair, the pair's chance to gain a territory is reduced by the factor $\delta \in[0.0,1.0]$. As the calculations of complete evolutionary vector fields is extremely time-consuming; we could do this for a number of selected cases only. We thus focus on scenarios covering a range of values of inbreeding depression $(\delta=0,0.4,0.6)$ for given values of breeding territories $N=10$, dispersal cost $\bar{c}=0.4$, and cost asymmetry $\Delta_{c}=0.1$ (comp. Fig. 2).

As long as inbreeding is less costly than dispersal, the system always settles into a state with dispersal biased in favor of males (Fig. 2a and b). Thus, in contradiction to the results of Gandon (1999), bi-stability vanishes completely as soon as $\delta \leq \bar{c}$. This holds already for rather small cost asymmetries $\left(\Delta_{c}\right)$. In accordance with Gandon (1999) we find completely biased dispersal (e.g. one sex disperses with a probability of one) when inbreeding is more costly than dispersal $(\delta>\bar{c})$ (Fig. 2c). However, the range of attraction for the two equilibria is clearly affected by the cost asymmetry. The ridge separating the two fields of attraction (dotted line in Fig. 2c) is shifted in favor of the sex with less dispersal cost $(c)$.

\section{Discussion}

Both, our numerical model and the individual-based simulations prove that differences in the cost of dispersal can lead to sex-biased dispersal. Other than with the magnitude of inbreeding depression there are no lower boundaries to the effect of cost asymmetries: even small differences are sufficient to induce sex-biased dispersal as long as the costs of dispersal do not become very small for both sexes. In contrast to Taylor (1988), Leturque 
and Rousset (2004), and Wild and Taylor (2004) we quantify that effect.

The results of the individual-based simulations agree well with the predictions from the numerical model as long as predicted dispersal probabilities (for both sexes) are considerably different from zero. Nonetheless, the evolved sex-bias consistently remained below the level predicted by the numerical model. This deviation should be the consequence of demographic stochasticity. In the numerical model the number of offspring is considered to be high enough so that the effects of demographic stochasticity can be ignored completely. Individual-based simulation experiments, on the other hand, take demographic fluctuations into account: the number of breeding pairs may fall below $N$, and population densities as well as sex-ratios will be variable across patches.

In these circumstances, avoidance of the risks of demographic stochasticity by bethedging (Hopper 1999) becomes a selective force favoring dispersal in both sexes. Additionally, with declining patch capacity, increasing variability in population size, or reduced dispersal, relatedness within patches increases, which will in turn enforce selection for dispersal in both sexes (Gandon 1999; Rousset and Ronce 2004). Consequently, in cases, where the numerical results predict females to be completely philopatric, we still get some female dispersal in the simulations of finite metapopulations (in agreement with Rousset and Ronce 2004). As the inclusive fitness balance of costs and benefits (comp. Equation 6 in the Appendix) also holds for the simulations, the amount of dispersal that females contribute to mean dispersal lessens selection on male dispersal and the sex-bias in dispersal is reduced. To check whether demographic stochasticity indeed contributes to selection for female dispersal, we ran additional individual-based simulations with lower $\lambda$ (increased stochasticity) and higher $\lambda$ (reduced stochasticity). If we set offspring number to a small value, i.e. $\lambda=4$, the deviation from the predicted sex-bias became more pronounced and female dispersal became higher than with $\lambda=10$ in the standard simulations. In reversal, the match between predicted sex-bias and that emerging in the simulations became closer if we raised offspring number to $\lambda=16$.

Concerning inbreeding, Gandon (1999) showed that the evolving sex-bias will only depend on starting conditions as inbreeding avoidance itself does not define which sex should be more dispersive. However, in our simulations we show that already a small bias in dispersal cost would favor dispersal of the sex with less costly dispersal, irrespective of starting conditions. Only when the effect of inbreeding depression becomes larger than the mean costs of dispersal, initial conditions determine the evolutionary trajectory; however, the "zone of attraction" becomes larger for the sex with lower costs of dispersal. Obviously, the magnitude of sex-bias in dispersal also becomes more pronounced if inbreeding depression and cost asymmetries interact.

Presumably, other types of asymmetries might also be responsible for the emergence of sex-biased dispersal. Obviously, this would be the case if the benefits of philopatry were different for the two sexes, e.g. because only offspring of one sex can inherit a territory from the parents. Another especially interesting case may be the effect of certain malekilling endoysymbionts, which lead to a difference in kin-structure for males and females and thus potentially promote sex-biased dispersal (Bonte et al. in press).

Direct evidence for gender-specific dispersal costs is rarely found in the literature. Ralls 
et al. (1980) and Holekamp (1984) report differential costs of dispersal in sperm whales respectively in ground squirrels. Also for species with non-overlapping generations, like insects, there are (to our knowledge) only few published results supporting an asymmetry in dispersal costs (Petit et al. 2001; Matter 2006; Rabasa et al. 2007). However, we assume that the lack of corresponding evidence is more due to the fact that it has rarely been looked for in empirical research. At least there are numerous arguments suggesting that sex-biased differences in dispersal ability or costs may be common. For example, body size is very likely to affect dispersal abilities - and many species show substantial differences in body size between the sexes. Differences in dispersal mortality may also arise because, at least in many insect species, females allocate resources into body mass and energy reserves for higher offspring production (del Castillo and Nunez-Farfan 2002), and there are often trade-offs between dispersal capacity and fertility in insects (Zera and Denno 1997; Marden 2000; Karlsson and Johansson 2008) or in some bird species (Kullberg et al. 2002). Consequently, dispersing females are often less fertile than philopatric individuals, and as dispersing females have to invest in both fecundity and dispersal ability they may pay higher costs for dispersal than males. There are also studies demonstrating higher mobility for males rather than for females, e.g. in the blue-winged grasshopper Oedipoda caerulescens (Maes et al. 2006). Generally, sexual selection for agility or strength, e.g. for resource or mate defense, could also increase dispersal ability and therefore reduce mortality during dispersal.

However, it may always remain difficult to distinguish between cause and effect in cases where a sexual bias in dispersal is observed: has one sex become more dispersive because it has been more successful in doing so, or has the more dispersive sex evolved a better ability to disperse after sex-biased dispersal emerged? Presumably, it is not even reasonable to try answering this question. Instead, in an evolutionary feedback, differences in dispersal ability and sex-bias are likely to jointly increase and to stabilize an evolutionary course which was originally catalyzed by another mechanism, e.g. inbreeding depression.

Acknowledgements - We are grateful for the financial support by the German Science Foundation (DFG PO 244/3-1).

\section{Appendix}

\section{Derivation of optimal dispersal probability for the numerical sim- ulations}

Gandon (1999) bases his model on the kin-selection model of Taylor (1988) which gives a condition for evolutionary stability for an unbiased dispersal strategy:

$$
c=R k,
$$

with $c$, the costs of dispersal, i.e. the probability to die during dispersal $(c \in[0 ; 1]), R$, the degree of relatedness in a population, and $k$, the probability to be a philopatric individual. 
This can be translated into a dispersal strategy by replacing $k$ with $(1-d) /(1-c d)$ :

$$
c=R \frac{1-d}{1-c d}
$$

with dispersal probability $d$. Equation 6 was also derived by Taylor (1988) and Gandon (1999). The left-hand side of Equation 6 represents the costs of dispersal and the righthand side describes the gain in inclusive fitness due to dispersal. By taking the risk of dying during dispersal $(c)$, a disperser reduces competition with $(1-d)(1-c d)$ philopatric individuals in its home patch, with an average relatedness to the disperser of $R$. In equilibrium, dispersal is tuned so that the costs of dispersal are balancing the benefits in inclusive fitness (see below).

$(1-d)$ is the proportion of philopatric individuals and $(1-c d)=1-d+(1-c) d$ describes the net flow of individuals for patches and denotes the proportion of the individuals alive after the dispersal phase. From this we get:

$$
d=\frac{R-c}{R-c^{2}}
$$

With sex-specific costs of dispersal and sex-specific dispersal, the coefficient of relatedness $R$ has to account for both aspects as $R$ itself depends on dispersal rates:

$$
R=\frac{1}{2 N-k_{1}-(N-1) / 2\left(2 k_{1}+k_{2}\right)^{2}}
$$

with

$$
\begin{aligned}
k_{1} & =\frac{\left(1-d_{m}\right)\left(1-d_{f}\right)}{\left(1-c_{m} d_{m}\right)\left(1-c_{f} d_{f}\right)} \\
k_{2} & =\frac{\left(1-d_{m}\right)\left(1-c_{f}\right) d_{f}+\left(1-d_{f}\right)\left(1-c_{m}\right) d_{m}}{\left(1-c_{m} d_{m}\right)\left(1-c_{f} d_{f}\right)}
\end{aligned}
$$

Following the approach of marginal gains in fitness (MGF) an expression for the ESS dispersal strategy for the sex-specific dispersal propensities $\left(d_{m}\right.$ for males and $d_{f}$ for females) can be obtained directly (comp. Gandon 1999). In the following we derive an expression for the ESS dispersal propensity $\left(d_{m, f}^{*}\right)$ under given (sex-specific) costs of dispersal ( $c_{m}$ for males and $c_{f}$ for females). Omitting the inbreeding depression included in Gandon's model and considering only diploid individuals, the MGF of dispersal $\left(G_{d}\right)$ and philopatry $\left(G_{p}\right)$ yield for males:

$$
\begin{aligned}
G_{p} & =P_{p}(1-k R), \\
G_{d} & =P_{d}\left(1-c_{m}\right),
\end{aligned}
$$

with $R$ as relatedness under offspring control of dispersal (see Eqn. 8), and 


$$
k=\frac{1-d_{m}}{1-c_{m} d_{m}}
$$

the probability to be a philopatric individual. $P_{p}$ and $P_{d}$ in Equations 9 and 10 are the probabilities of mating success of philopatric and dispersing males which (omitting inbreeding depression again) are calculated as

$$
P_{p}=P_{d}=\frac{1}{1-c_{m} d_{m}}
$$

The evolutionary stable dispersal probability equalizes the MGF from dispersal and philopatry, so that from $G_{d}=G_{p}$ we can derive from Equations 9 and 10 and by using Equation 12:

$$
1-\frac{1-d_{m}}{1-c_{m} d_{m}} R=1-c_{m}
$$

From this follows:

$$
\begin{aligned}
\frac{1-d_{m}}{1-c_{m} d_{m}} R & =c_{m}, \\
\Rightarrow\left(1-d_{m}\right) R & =c_{m}-c_{m}^{2} d_{m}, \\
\Rightarrow R-d_{m} R & =c_{m}-c_{m}^{2} d_{m}, \\
\Rightarrow R-c_{m} & =d_{m} R-c_{m}^{2} d_{m}, \\
\Rightarrow \frac{R-c_{m}}{R-c_{m}^{2}} & =d_{m},
\end{aligned}
$$

with $R>c_{m}$. By letting Equation 14 equilibrate in a numerical simulation we arrive at evolutionary stable values for $d_{m}^{*}$. The evolutionary stable female dispersal strategy can be obtained by changing suffix $m$ to $f$ and thereby we arrive at:

$$
\begin{aligned}
d_{m}^{*} & =\frac{R^{*}-c_{m}}{R^{*}-c_{m}^{2}}, \\
d_{f}^{*} & =\frac{R^{*}-c_{f}}{R^{*}-c_{f}^{2}} .
\end{aligned}
$$




\section{References}

Bonte, D., Hovestadt, T., Poethke, H., in press. Male-killing endosymbionts: influence of environmental conditions on persistence of host metapopulation. BMC Evolutionary Ecology.

Bowler, D. E., Benton, T. G., 2005. Causes and consequences of animal dispersal strategies: relating individual behaviour to spatial dynamics. Biological Reviews of the Cambridge Philosophical Society (London) 80 (2), 205-225.

Clarke, A. L., Saether, B. E., Roskaft, E., 1997. Sex biases in avian dispersal: A reappraisal. Oikos 79 (3), 429-438.

Clobert, J., Danchin, E., Dhont, A. A., Nichols, J. D. (Eds.), 2001. Dispersal. Oxford University Press, New York.

del Castillo, R. C., Nunez-Farfan, J., 2002. Female mating success and risk of prereproductive death in a protandrous grasshopper. Oikos 96 (2), 217-224.

Gandon, S., Oct. 1999. Kin competition, the cost of inbreeding and the evolution of dispersal. Journal of Theoretical Biology 200 (4), 345-364.

Holekamp, K. E., 1984. Natal dispersal in belding's ground squirrels (Spermophilus beldingi). Behavioral Ecology and Sociobiology 16, 21-30.

Hopper, K. R., 1999. Risk-spreading and bet-hedging in insect population biology. Annual Review of Entomology 44 (1), 535-560.

Hovestadt, T., Nieminen, M., in press. Costs and benefits of dispersal in butterflies. In: Settele, J., Komvicka, M., Shreeve, T. G., Dyck, H. V. (Eds.), Ecology of butterflies in Europe. Cambridge University Press, Cambridge, pp. 0-0.

Ims, R. A., Hjermann, D. O., 2001. Condition-dependent dispersal. In: Clobert, J., Danchin, E., Dhont, A. A., Nichols, J. D. (Eds.), Dispersal. Oxford University Press, Oxford, New York, pp. 203-216.

Karlsson, B., Johansson, A., Sep 2008. Seasonal polyphenism and developmental tradeoffs between flight ability and egg laying in a pierid butterfly. Proceedings of the Royal Society B: Biological Sciences 275 (1647), 2131-2136.

Kullberg, C., Houston, D. C., Metcalfe, N. B., 2002. Impaired flight ability-a cost of reproduction in female blue tits. Behavioral Ecology 13 (4), 575-579.

Lawson Handley, L. J., Perrin, N., 2007. Advances in our understanding of mammalian sex-biased dispersal. Molecular Ecology 16 (8), 1559-1578.

Leturque, H., Rousset, F., 2003. Joint evolution of sex ratio and dispersal: conditions for higher dispersal rates from good habitats. Evolutionary Ecology 17 (1), 67-84.

Leturque, H., Rousset, F., 2004. Intersexual competition as an explanation for sex-ratio and dispersal biases in polygynous species. Evolution 58 (11), 2398-2408. 
Maes, D., Ghesquiere, A., Logie, M., Bonte, D., 2006. Habitat use and mobility of two threatened coastal dune insects: implications for conservation. Journal of Insect Conservation 10 (2), 105-115.

Marden, J. H., 2000. Variability in the size, composition, and function of insect flight muscles. Annual Review of Physiology 62 (1), 157-178.

Matter, S., 2006. Changes in landscape structure decrease mortality during migration. Oecologia 150 (1), 8-16.

Matthysen, E., 2005. Density-dependent dispersal in birds and mammals. Ecography 28 (3), 403-416.

Motro, U., 1991. Avoiding inbreeding and sibling competition: The evolution of sexual dimorphism for dispersal. American Naturalist 137 (1), 108-115.

Motro, U., 1994. Evolutionary and continuous stability in asymmetric games with continuous strategy sets - the parental investment conflict as an example. American Naturalist 144 (2), 229-241.

Parvinen, K., Dieckmann, U., Gyllenberg, M., Metz, J. A. J., 2003. Evolution of dispersal in metapopulations with local density dependence and demographic stochasticity. Journal of Evolutionary Biology 16 (1), 143-153.

Perrin, N., Mazalov, V., 1999. Dispersal and inbreeding avoidance. American Naturalist 154, 282-292.

Perrin, N., Mazalov, V., 2000. Local competition, inbreeding, and the evolution of sexbiased dispersal. American Naturalist 155 (1), 116-127.

Petit, S., Moilanen, A., Hanski, I., Baguette, M., 2001. Metapopulation dynamics of the bog fritillary butterfly: movements between habitat patches. Oikos 92 (3), 491-500.

Poethke, H. J., Hovestadt, T., 2002. Evolution of density- and patch-size-dependent dispersal rates. Proceedings of the Royal Society B: Biological Sciences 269 (1491), 637645 .

Poethke, H. J., Pfenning, B., Hovestadt, T., 2007. The relative contributions of individualand kin-selection in the evolution of density-dependent dispersal rates. Evolutionary Ecology Research 9, 41-50.

Prugnolle, F., de Meeus, T., 2002. Inferring sex-biased dispersal from population genetic tools: a review. Heredity 88, 161-165.

Rabasa, S. G., Gutierrez, D., Escudero, A., 2007. Metapopulation structure and habitat quality in modelling dispersal in the butterfly Iolana iolas. Oikos 116 (5), 793-806.

Ralls, K., Brownell, R. L., Ballou, J., 1980. Differential mortality by sex and age and mammals, with special reference to the sperm whale. In: Donovan, G. P. (Ed.), Report of the International Whaling Commission (Special Issue). Vol. 2. pp. 233-243. 
Ronce, O., 2007. How does it feel to be like a rolling stone? Ten questions about dispersal evolution. Annual Review of Ecology, Evolution, and Systematics 38 (1), 231-253.

Rousset, F., Ronce, O., 2004. Inclusive fitness for traits affecting metapopulation demography. Theoretical Population Biology 65, 127-141.

Taylor, P., 1988. An inclusive fitness model for dispersal of offspring. Journal of Theoretical Biology 130 (3), 363-378.

Wild, G., Taylor, P. D., 2004. Kin selection models for the co-evolution of the sex ratio and sex-specific dispersal. Evolutionary Ecology Research 6 (4), 481-502.

Wolfram Research, Inc., 1999. Mathematica Edition: Version 4.0. Wolfram Research, Inc., Champaign, Illinois.

Zera, A. J., Denno, R. F., 1997. Physiology and ecology of dispersal polymorphism in insects. Annual Review of Entomology 42, 207-230. 